EPJ Web of Conferences 59, 04001 (2013)

DOI: $10.1051 /$ epjconf/20135904001

(C) Owned by the authors, published by EDP Sciences, 2013

\title{
Integrated thermodynamic model for ignition target performance
}

\author{
P.T. Springer ${ }^{1}$, C. Cerjan ${ }^{1}$, R. Betti ${ }^{4}$, J.A. Caggiano ${ }^{1}$, M.J. Edwards ${ }^{1}$, \\ J.A. Frenje ${ }^{3}$, V.Yu. Glebov ${ }^{4}$, S.H. Glenzer ${ }^{1}$, S.M. Glenn ${ }^{1}$, N. Izumi ${ }^{1}$, O. Jones ${ }^{1}$, \\ G. Kyrala ${ }^{2}$, T. Ma ${ }^{1}$, J. McNaney ${ }^{1}$, M. Moran ${ }^{1}$, D.H. Munro ${ }^{1}$, S. Regan ${ }^{4}$, \\ T.C. Sangster ${ }^{4}$, S. Sepke ${ }^{1}$, H. Scott ${ }^{1}$, R.P.J. Town ${ }^{1}$, S.V. Weber ${ }^{1}$ and B. Wilson ${ }^{1}$ \\ ${ }^{1}$ LLNL, Livermore, CA, USA \\ ${ }^{2}$ LANL Los Alamos, NM, USA \\ ${ }^{3}$ MIT, Cambridge, Ma, USA \\ ${ }^{4}$ Laboratory for Laser Energetics, Rochester, NY, USA
}

\begin{abstract}
We have derived a 3-dimensional synthetic model for NIF implosion conditions, by predicting and optimizing fits to a broad set of x-ray and nuclear diagnostics obtained on each shot. By matching $\mathrm{x}$-ray images, burn width, neutron time-of-flight ion temperature, yield, and fuel $\rho$ r, we obtain nearly unique constraints on conditions in the hotspot and fuel in a model that is entirely consistent with the observables. This model allows us to determine hotspot density, pressure, areal density $(\rho r)$, total energy, and other ignition-relevant parameters not available from any single diagnostic. This article describes the model and its application to National Ignition Facility (NIF) tritium-hydrogen-deuterium (THD) and DT implosion data, and provides an explanation for the large yield and $\rho$ r degradation compared to numerical code predictions.
\end{abstract}

To optimize the target and laser parameters for ignition [1, 2], it is important to understand the implosion conditions achieved in a given experiment, independent of the design predictions. To this end, we developed and applied a model to estimate the performance of an implosion and assess temperature, density, and composition distributions within the assembled core, solely from the experimental data taken during the shot. Using radiative, equation of state, nuclear fusion relations for relevant materials, and an approximation of pressure equilibrium within the hotspot [3], we derive a 3-dimensional representation of the capsule density and temperature profiles at stagnation, by predicting and optimizing fits to a broad set of x-ray and nuclear diagnostics. This model allows us to determine hotspot density, pressure, areal density $(\rho r)$, total energy, and other ignition-relevant parameters not available from any single diagnostic. This approach has been validated by comparing results with radiation-hydrodynamic simulations and has produced semi-quantitative (10-20\%) agreement.

We begin by constructing a 3D model for the temperature and density conditions of the imploded core at neutron bang time, defined as the time of peak neutron and $10-20 \mathrm{keV} x$-ray production in the capsule [4]. We further assume that this imploded core, including both hotspot and fuel, is at a uniform pressure $\mathrm{P}_{\mathrm{hs}}$. So for a given density profile $\rho(\mathrm{r}, \theta, \phi)$, we derive an associated temperature profile using the hydrogenic equation-of-state, which includes effects of Fermi degeneracy in the dense shell [5]. This description produces a relatively complete characterization of the implosion temperature and density distributions, and from this we can calculate the complete properties of the x-ray emission and nuclear fusion processes to predict the ensemble of diagnostic signatures from the implosion.

Given an assumed pressure and density profile, we calculate the waist and polar x-ray emission images, using a free-free emissivity derived from the VISTA opacity model, and a Detailed

This is an Open Access article distributed under the terms of the Creative Commons Attribution License 2.0, which permits unrestricted use, distribution, and reproduction in any medium, provided the original work is properly cited. 

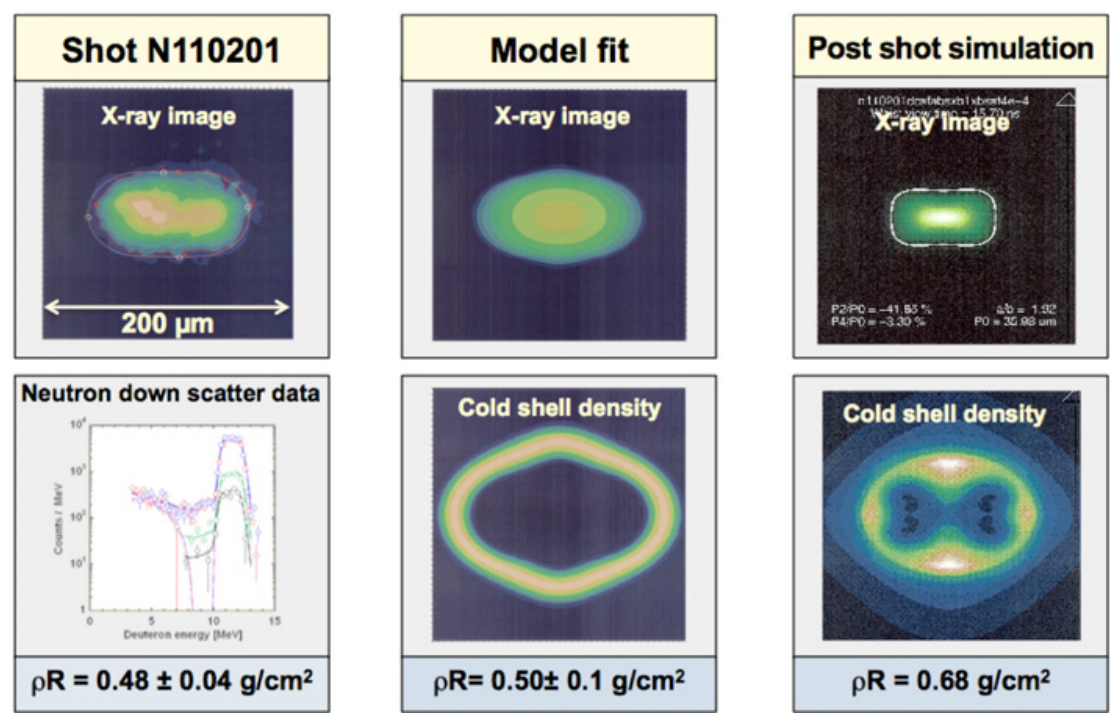

Figure 1. For Shot N110201 the model produces a very good match to x-ray images, Fuel $\rho$ r, Yield and Tion (not shown). The post-shot simulations predict greater convergence, Fuel $\rho \mathrm{r}$, and Yield.

Configuration Accounting, DCA, atomic model for non-equilibrium Ge impurity emission [6]. The $\mathrm{x}$-ray emission is proportional to $\rho(\mathrm{r}, \theta, \phi)^{2}(\mathrm{~h} v)^{-.33} \exp (-\mathrm{h} v / \mathrm{T})$, and so for a given pressure the $\mathrm{x}$-ray emission profile can be expressed solely as a function of density. While the size of the experimental $\mathrm{x}$-ray images determines the volume of the hot core region, the $\mathrm{x}$-ray emission profile determines the density profile. We do not use the absolute intensity of the x-ray images.

Similarly, analytic fits to light ion thermonuclear fusion rates are well known from stellar astrophysical research [7]. From an assumed pressure and density profile, one can calculate the reactivity, and using the Brysk formula, a neutron spectrum as a function of neutron energy, integrated over the hotspot, which contains a distribution of temperature and burn rates [8]. The thermal distribution results in a dispersion of the neutron arrival times at a distant detector, and can be compared with the nTOF signal to provide an average temperature for the burn region [9]. Integrating the fusion reactivity over the hotspot and multiplying by the burn width $\tau_{\mathrm{b}}$ gives the total yield, which is measured better than $10 \%$ absolutely. Thus the yield is then used to determine the absolute density, not simply its profile.

For a given density profile, using the method discussed above, one generates a prediction of model data, which are compared with the actual data. Figure 1 shows an example for THD Shot N110201 of our model fit to the ensemble of observed data including x-ray images, yield, Tion, fuel $\rho$ r, and total fuel mass. To obtain this match, the density profile is varied by specifying a set of parameters, typically 10 20 , that describe the volume, profile, and peak temperature of the lower density central hotspot in which the yield is produced, as well as the radial position (30-100 microns), peak density (30-1200 g/cc), and thickness (5-20 microns), including both polar and azimuthal angle variations, of the cooler dense fuel surrounding the hotspot. A multivariate chi-squared minimization algorithm [10] is then used to determine the implosion conditions' $\rho(\mathrm{r}, \theta, \phi)$ and $\mathrm{P}_{\mathrm{hs}}$ (and associated uncertainties), that best match the complete data set.

We performed a "blind" validation study in which implosion metrics were derived solely using simulated neutron and x-ray data, as described above, and compared to the same implosion metrics derived directly from the same simulation. Results of such tests are summarized in table 1, showing that model is typically better than $10 \%$. There is a total estimated uncertainty of order $20 \%$ due to the precision of the data used. 
Table 1. Results of a "blind" validation study showing model accuracy of order $10 \%$.

\begin{tabular}{|l|c|c|c|}
\hline $\begin{array}{l}\text { Implosion } \\
\text { Metric }\end{array}$ & Simulation & Model Fit & $\begin{array}{c}\text { Relative } \\
\text { Error\% }\end{array}$ \\
\hline $\mathrm{Ti}(\mathrm{keV})$ & 3.20 & 3.42 & 7 \\
\hline$\rho_{\text {lon }}\left(\mathrm{g} / \mathrm{cm}^{3}\right)$ & 23.9 & 24.0 & 1 \\
\hline Mass $(\mu \mathrm{g})$ & 18.9 & 20.2 & 6 \\
\hline Volume $\left(\mu \mathrm{cm}^{3}\right)$ & 50.3 & 50.3 & 1 \\
\hline Energy $(\mathrm{kJ})$ & 6.54 & 6.48 & -1 \\
\hline $\mathrm{P}(\mathrm{Gbar})$ & 69.8 & 68.0 & -1 \\
\hline Yield $10^{11}$ & 1.05 & 1.07 & 2 \\
\hline
\end{tabular}

Table 2. Implosion metrics for NIF THD and DT implosions compared with typical post-shot simulation.

\begin{tabular}{|l|c|c|c|c|c|}
\hline Implosion Metric & $\begin{array}{c}\text { Typical } \\
\text { Post-shot } \\
\text { Simulation }\end{array}$ & $\begin{array}{c}\text { THD N100929 } \\
(\mathbf{1 M J}, \text { Rev 5) } \\
\text { Model (expt) }\end{array}$ & $\begin{array}{c}\text { THD N110201 } \\
(\mathbf{1 . 2 M J} 2 \times \text { foot) } \\
\text { Model }(\mathbf{e x p t})\end{array}$ & $\begin{array}{c}\text { THD N110212 } \\
(\mathbf{1 . 2 M J} 1 \times \text { Foot) } \\
\text { Model }(\mathbf{e x p t})\end{array}$ & $\begin{array}{c}\text { DT N110620 } \\
(\mathbf{1 . 4 M J )} \\
\text { Model }(\mathbf{e x p t})\end{array}$ \\
\hline Yield $10^{14}$ & 31 & 0.46 & 6.4 & 7.5 & 4.5 \\
\hline Ti $(\mathrm{keV})$ & 3.2 & 2.45 & 3.5 & 3.9 & 4.3 \\
\hline Hotspot Energy $(\mathrm{kJ})$ & 6.2 & 3.7 & 5.5 & 3.2 & 1.3 \\
\hline $\mathrm{P}(\mathrm{Gbar})$ & 165 & 9.4 & 19 & 31 & 80 \\
\hline Volume $\left(\mu \mathrm{cm}^{3}\right)$ & 0.25 & 2.6 & 1.9 & 0.7 & 0.1 \\
\hline $\mathrm{P} \rho^{-5 / 3}$ & 0.095 & 0.538 & 0.861 & 0.703 & 0.358 \\
\hline Density $\left(\mathrm{g} / \mathrm{cm}^{3}\right)$ & 88 & 5.6 & 6.4 & 9.7 & 26 \\
\hline Mass $(\mu \mathrm{g})$ & 22 & 29 & 10 & 6 & 2.8 \\
\hline
\end{tabular}

The model was then applied to predict performance of NIF THD and DT data, and used to derive key performance metrics, a few of which are summarized in table 2. The yield and inferred pressure are 5-20 times lower than post-shot simulation, and these differences are much larger than 20\% estimated uncertainty in the analysis. The total energy of the fuel and hotspot are mostly consistent with the expected implosion energy (except for the DT shot) but the hotspot entropy or adiabat is found to be much higher in the data than the simulation. The fuel $\rho \mathrm{r}$ is consistent with the bulk of the fuel having a nominal adiabat, but a reduced $\rho$ r simply because of the limited total mass, and larger than expected hotspot.

In addition to these metrics for the hotspot, the model also has constraints on the cold fuel provided by the $\rho$ r, total mass, and the hotspot volume and pressure. The model predicts the total energy in the implosion as well as the adiabat of the cold fuel, and these correspond to nominal values. It is useful to plot the stagnation pressure and inferred hotspot volume on a PV diagram as illustrated in Fig. 2. At stagnation the implosions will lie on a locus of points in the PV diagram determined by the implosion energy $\left(\mathrm{PV} \sim \mathrm{mv}^{2}\right.$ ), and the implosion adiabat $\left(\mathrm{PV}^{5 / 3} \sim\right.$ const). The dashed red lines represent the hotspot energy for the different implosion velocities in the experiments, which match those of the Rev 5 point design. For a given energy of the hotspot, the pressure is determined by the implosion adiabat $\left(\mathrm{PV}^{5 / 3} \sim\right.$ const), which is set by the energy and volume of the hotspot at peak velocity when it begins to decelerate the shell. The dashed blue lines represent possible implosion adiabats, one for the point design, and one that matches N110212. The implosion performance is degraded because the hot spot is on too high an adiabat. This implies that the volume at peak velocity is 5 times greater than the design goal, and occurs at a radius of $233 \mu \mathrm{m}$, rather than the simulated $133 \mu \mathrm{m}$. This could occur with a higher than desired velocity for the first few microns of mass in the inner ice layer, which stagnate early and the stagnation shock arrives at the shell at a larger radius. Other consequences of this would be an 


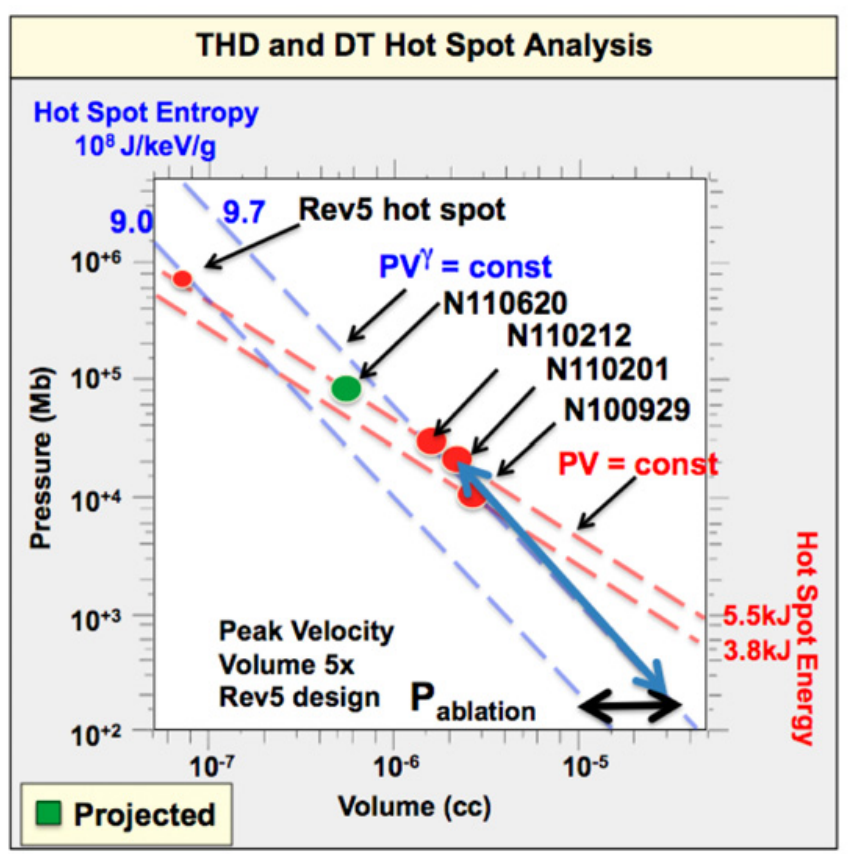

Figure 2. A plot of the stagnation pressure and volume for THD and DT shots compared with the Rev5 design.

early shock flash, higher than expected temperature, lower than expected pressure, yield and $\rho$ r. All of these trends are observed in capsule implosions. A stronger than expected $5^{\text {th }}$ shock or significant shock mistiming (from LEH window ice) is the most likely cause of the problem. The first DT shot 110620 also shows a significantly lower than expected hotspot energy.

This work was performed under the auspices of the U.S. Department of Energy by Lawrence Livermore National Security, LLC, Lawrence Livermore National Laboratory under Contract DE-AC52-07NA27344.

\section{References}

[1] T. R. Dittrich et al., Phys. Plasmas 6, 2124 (1999)

[2] J. D. Lindl, "Inertial Confinement Fusion,” (Springer-Verlag, New York, 1998)

[3] M. C. Herrmann, M. Tabak, J. D. Lindl, Nucl. Fusion 41, 99 (2001); R. Betti et al., Phys. Plasmas, 8, 5257 (2001); J. Meyer-ter-Vehn, Nucl. Fusion 22, 5619 (1982); V. A. Smalyuk, et al., Phys. Plasmas, 12, 052706 (2005)

[4] P. T. Springer and C. Cerjan, LLNL internal memorandum (2010)

[5] Report on the Los Alamos Equation-of-State Library. T-4 group, Los Alamos National Laboratory, Los Alamos, N. M. Report LALP-83-4

[6] H.A. Scott, private communication

[7] G.R. Coughlan and W.A. Fowler, Atomic Nucl. Data 40, 283 (1988)

[8] H. Brysk, Plasma Physics 15, 611 (1973)

[9] V. Glebov et al., Rev. Sci Instrum. 77, 10E715 (2006)

[10] W. H. Press et al., "Numerical Recipes," Section 14.3 (Cambridge University Press, Cambridge, 1986) 